\title{
Narrative review: predicting future molecular and clinical profiles of prostate cancer in the United States
}

\author{
Matteo Santoni ${ }^{1}$, Alessia Cimadamore ${ }^{2}$, Francesco Massari ${ }^{3}$, Giulia Sorgentoni ${ }^{1}$, Liang Cheng $^{4}$, \\ Antonio Lopez-Beltran ${ }^{5}$, Nicola Battelli ${ }^{1}$, Rodolfo Montironi ${ }^{2}$ \\ ${ }^{1}$ Oncology Unit, Macerata Hospital, Macerata, Italy; ${ }^{2}$ Section of Pathological Anatomy, Polytechnic University of the Marche Region, School of \\ Medicine, United Hospitals, Ancona, Italy; ${ }^{3}$ Medical Oncology, IRCCS Azienda Ospedaliero-Universitaria di Bologna, Bologna, Italy; ${ }^{4}$ Department \\ of Pathology and Laboratory Medicine, Indiana University School of Medicine, Indianapolis, IN, USA; ${ }^{5}$ Department of Surgery, Cordoba University \\ Medical School, Cordoba, Spain \\ Contributions: (I) Conception and design: M Santoni, A Cimadamore, R Montironi; (II) Administrative support: R Montironi; (III) Provision of study \\ materials or patients: M Santoni, F Massari, G Sorgentoni; (IV) Collection and assembly of data: M Santoni, G Sorgentoni; (V) Data analysis and \\ interpretation: A Lopez-Beltran, L Cheng, N Battelli; (VI) Manuscript writing: All authors; (VII) Final approval of manuscript: All authors. \\ Correspondence to: Matteo Santoni, MD. Oncology Unit, Macerata Hospital, via Santa Lucia 2, 62100, Macerata, Italy. Email: mattymo@alice.it.
}

\begin{abstract}
Prostate cancer represents the most frequent tumor in men, accounting for the $21 \%$ of all diagnosed tumors, with 191,930 new cases and 33,330 deaths estimated in 2020. Advanced prostate cancer represents a heterogeneous disease, ranging from hormone naive or hormone sensitive to castration resistant. The therapeutic armamentarium for this disease has been implemented in the last years by novel hormonal therapies and chemotherapies. However, the percentage of patients who achieve complete responses still results negligible. On this scenario, the design of clinical trials investigating new therapeutic approaches represent a dramatic medical need. Predicting cancer incidence may be fundamental to design specific clinical trials, to optimize the allocation of economic resources, and to plan future cancer control programs. $E R G, S P O P$ and DDR genes alterations can act as therapeutic targets in prostate cancer patients and can be tested to identify a gene-selected patient population to enrol in specific trials. According to our predictions, $E R G$ gene fusions will be the most predominant molecular subtype, accounting for 69,050 new cases in 2030. Mutation in SPOP gene will be diagnosed in 16,512 tumors, corresponding to the number of cases associated with alterations in DDR genes (including 7,956 BRCA2 mutated tumors). In this article, we analyzed and discussed the future molecular and clinical profiles of prostate cancer in the United States, aimed to describe a series of distinct subpopulations and to quantify potential clinical trial candidates in the next years.
\end{abstract}

Keywords: BRCA2; castration resistance; DNA damage repair (DDR); future tumor burden; genetic alterations; prostate cancer

Submitted Nov 19, 2020. Accepted for publication Jan 21, 2021.

doi: $10.21037 /$ tau-20-1439

View this article at: http://dx.doi.org/10.21037/tau-20-1439

\section{Introduction}

Cancer is the second most lethal disease condition in the United States, with more than 600,000 deaths in 2020 (1). While the number of estimated new cases has overcome 1.8 million in 2020, the rate of cancer-related deaths has declined due to the development of more effective diagnostic and therapeutic approaches (1). Prostate cancer represents the most frequent tumor in men, accounting for the $21 \%$ of all diagnosed tumors, with 191,930 new cases and 33,330 deaths estimated in 2020 (1). The incidence of prostate cancer is about $60 \%$ higher in blacks than in whites $(1,2)$. Smoking attitude (3) and obesity $(4,5)$ have been recognized as negative prognostic factors associated with aggressive and fatal prostate cancer. Strong familial predisposition may be found in $5-10 \%$ of prostate cancers (1). 
Advanced prostate cancer represents a heterogeneous disease, ranging from hormone naive or hormone sensitive to castration resistant, both containing patients who have demonstrable metastatic and non-metastatic states. Earlystage prostate cancer usually presents without symptoms. More advanced disease stages are characterized by a series of clinical manifestation such as urinary symptoms (i.e., interrupted urine flow, burning and pain), fatigue, hematologic alterations and bone pain. Prostate cancer includes a variety of clinical entities that range from indolent forms to aggressive variants characterized by rapidly progressive disease course (6). The complexity of tumor environment is related to a series of factors including the heterogeneity between primary tumor and metastases (7) and marked alterations of cell metabolism (8).

Predicting cancer incidence may be fundamental to design specific clinical trials, to optimize the allocation of economic resources, and to plan future cancer control programs. This has become essential considering the rising costs of the most recently oncologic drugs approved by the US Food and Drug Administration (FDA) in the last decades (9-11). Recently, we published the results of an analysis by Artificial Neural Networks (ANNs) aimed to predict the incidence of breast, colorectal, lung and prostate cancer in the United States (12). We reported a decreasing trend in terms of incidence of prostate cancer of from 1990 to 2050. In particular, our predictions showed that prostate cancer incidence would decrease from 200 cases/100,000 habitants registered in the 1990s in USA to less than 90/100,000 from 2020. Furthermore, our algorithm registered different decreasing trends among races/ethnicities, which were similar to overall population in white and black patients, while Asian/ Pacific ethnicity as well as American Indian and Alaska native were characterized by a lower incidence (12).

In this article, we analyzed and discussed the future profiles of prostate cancer in the United States, aimed to describe a series of distinct subpopulations and to quantify potential clinical trial candidates in the next years. A PubMed search using the keywords "Future tumor burden", "Genetic alterations", "Prostate Cancer" from January 2005 to October 2020 was performed. We present the following article in accordance with the Narrative Review reporting checklist (available at http://dx.doi.org/10.21037/tau-20-1439).

\section{Narrative review}

\section{Tumor genetic landscape}

According to our predictions (12), the number of new cases of prostate cancer will account for 191,486 in 2020 and will decrease to 150,110 in 2030 , reaching 99,313 patients in 2050. Comprehensive molecular profiling of prostate cancer has helped the identification of distinct subtypes and driving alterations $(13,14)$. In 2015, the Cancer Genome Atlas Research Network presented a comprehensive molecular analysis of 333 primary prostate carcinomas (15). They showed that $74 \%$ of tumors were assignable to seven distinct molecular classes: tumors harbouring fusions involving ETSrelated gene (ERG,46\%), ETS-translocation variant 1 (ETV1, $8 \%)$, ETS-translocation variant 4 (ETV4, 4\%) or Friend Leukemia Integration 1 (FLI1, 1\%) or mutations in Speckle-Type POZ Protein (SPOP, 11\%), Forkhead box protein A1 (FOXA1, $3 \%)$ and Isocitrate Debydrogenase 1 (IDH1, 1\%) (15).

Furthermore, a growing body of evidences suggest that germline and somatic mutations on DNA repair genes should be investigated in patients with prostate cancer due to the development of PolyAdenosine Diphosphate (ADP)Ribose Polymerase (PARP) inhibitors (16). It has been estimated that alterations in DNA Damage Repair (DDR) genes can be found in approximately $11 \%$ of prostate cancers (17).

According to our predictions, ERG gene fusions will be the most predominant molecular subtype, accounting for 69,050 new cases in 2030. Mutation in SPOP gene will be diagnosed in 16,512 tumors, corresponding to the number of cases associated with alterations in DDR genes (including 7,956 BRCA2 mutated tumors). The future distribution of new cases of prostate cancer in the United States according to tumor driving alterations is reported in Figure 1.

These data support the role of ERG, SPOP and DDR alterations as therapeutic targets in patients with prostate cancer and underline the possibility of designing clinical trials enrolling only specific gene-tested subpopulations.

\section{Metastatic hormone-sensitive prostate cancer (mHSPC)}

It has been estimated that biochemical recurrence, a condition defined as rising prostate-specific antigen (PSA) levels following local therapy, occurs in about $30 \%$ of patients with prostate cancer (18). On the other hand, mHSPC is defined by the presence of metastasis in patients who have not yet received, or are continuing to respond to, hormone therapy. mHSPC represents about $15 \%$ of annual prostate cancer incidence in the United States (19). The therapeutic approach to $\mathrm{mHSPC}$ has been completely changed by the evidence that chemotherapy with docetaxel (20) and abiraterone acetate (21) demonstrated an advantage in terms of OS in patients with high-risk or high-volume prostate cancer 


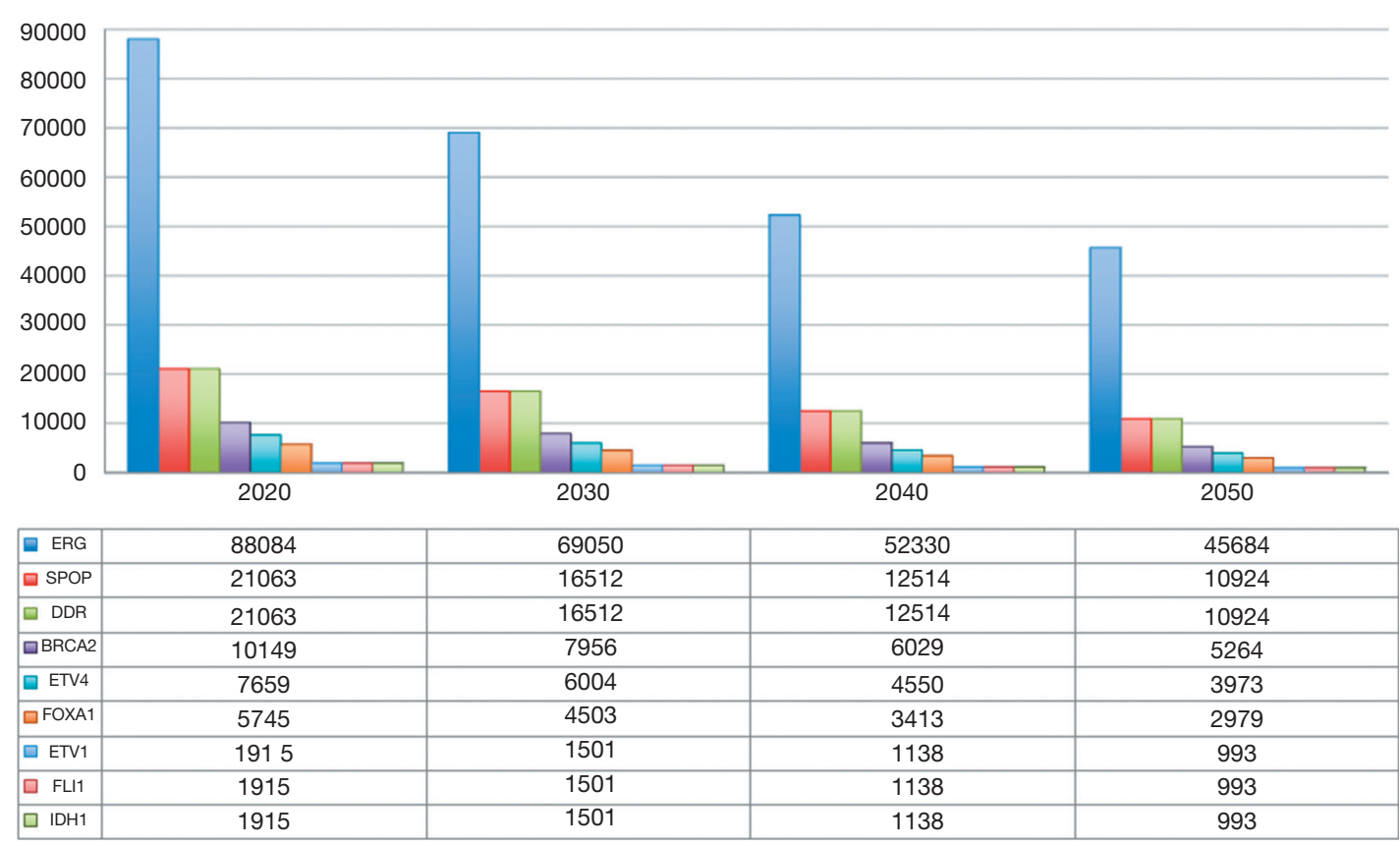

Figure 1 Number of new cases of prostate cancer and distribution according to tumor driving alterations in the United States in the next 30 years.

metastatic at diagnosis. In the same view, enzalutamide (22) and apalutamide (23) demonstrated to be effective in patients with both metastatic disease at diagnosis and recurrence after primary treatment, thus increasing the number of candidate to novel hormone therapies.

Under a clinical and pharmacoeconomic point of view, predicting the number of mHSPC patients will be very useful to understand the future therapeutic landscape of this disease. According to our estimates, mHSPC will be diagnosed in 28,723 patients in 2020 , decreasing to 22,516 in 2030 till 14,897 in 2050 (Figure 2).

\section{Non-metastatic castration resistant prostate cancer}

Despite several hormonal therapies being available at present for the management of metastatic prostate cancer, the natural history of this tumor leads inexorably to the development of resistance to hormone inhibition. Many genetic and epigenetic mechanisms are associated with castration resistance: (I) restored androgen receptor (AR) signaling, (II) AR bypass signaling, and (III) complete AR independence (24).

The standard imaging modalities to define the presence of regional or distant metastasis or the different resistant mechanisms to the available treatments are still an issue of debate. Non-metastatic castration resistant prostate cancer is defined by the absence of distant metastases at TC scan and bone scintigraphy associated with resistance to hormone therapy. New hormonal agents apalutamide $(25,26)$, enzalutamide $(27,28)$ and darolutamide $(25,29)$ have completely changed the prognosis of patients with non-metastatic castration-resistant prostate cancer (30). In 2019, Di Nunno and his group performed a meta-analysis aimed at estimating the pooled effect of new hormonal compounds in terms of metastasis-free survival (MFS) and overall survival (OS) in overall and specific subpopulations of prostate cancer (30). They showed that the advantage in terms of MFS and OS of these three agents were significant in all subgroups. Apalutamide was associated to a higher risk to develop falls, fractures and rash, while enzalutamide and darolutamide resulted in a higher risk of cardiovascular toxicity (31).

Non-metastatic castration-resistant disease accounts for approximately $10 \%$ of hormone-sensitive prostate cancers (32). Based on these data, we can estimate a total of 2,872 patients in 2020 and a reduction to 2,252 in 2030 (Figure 2).

\section{Metastatic castration resistant prostate cancer}

In the last decade, targeting persistent AR activity by newgeneration hormonal therapies abiraterone acetate and enzalutamide has completely changed the outcome of patients with metastatic castration-resistant prostate cancer, increasing 


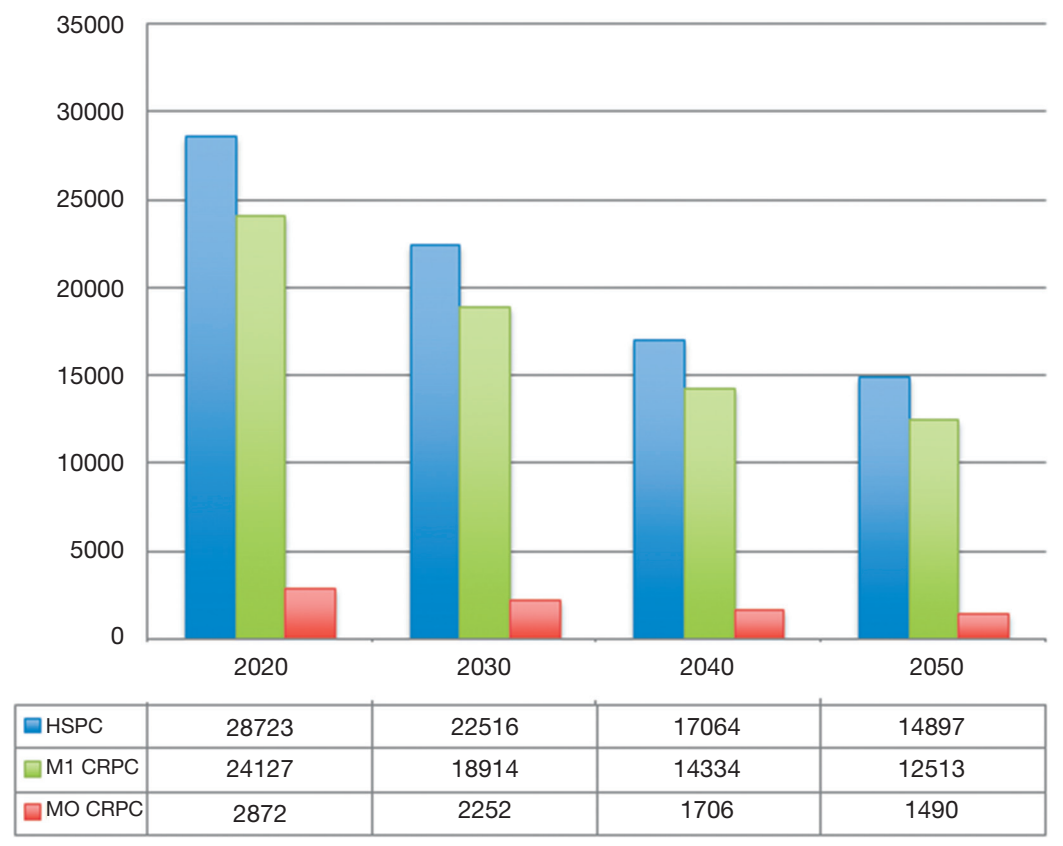

Figure 2 Number of new cases of prostate cancer and distribution according to castration resistance and metastasis the United States in the next 30 years in.

patients survival and quality of life (24). The standard imaging techniques to assess the presence of regional or distant metastasis or the different mechanisms of drug resistance are still an issue of debate. The choice between chemotherapy and new-generation hormonal therapies are mainly based on the presence of symptomatic disease, which can be preferably treated with chemotherapy in docetaxel-fit patients, while asymptomatic or paucisymptomatic patients can be preferably treated with enzalutamide or abiraterone acetate. Radium-223 is also a therapeutic option in docetaxelunfit patients or already pretreated with both docetaxel and enzalutamide/abiraterone acetate.

It has been reported that $84 \%$ of patients have metastases at time of castration resistant prostate cancer, while $33 \%$ of patients without metastases at diagnosis will develop them within 2 years (33). As reported in Figure 2, the number of patients with castration resistant disease can be estimated at 24,127 in 2020 and will reach 18,914 in 2030 (Figure 2).

\section{Future directions}

Predicting future cancer burden may represent an effective tool to optimize the allocation of economic resources and to plan future cancer control programs. To increase the accuracy of predicting models for cancer incidence, investigating the risk factors associated with tumor development is absolutely fundamental. Age, ethnicity, tobacco consumption and obesity are associated with the risk of developing prostate cancer during life-time. However, a series of factors, including alteration in host immune cells and gut microbiota, is emerging and should be taken into account in the context of future predicting models.

Prostate cancer is a heterogeneous disease with a variable natural history. Despite the long-term survival of earlystage prostate cancer, metastatic disease remains still incurable. The lethality of prostate cancer is due to the lack of durable drug responses on the scenario of extreme tumor heterogeneity on both genetic and cell biological levels. A better comprehension of the molecular oncogenic drivers of prostate carcinogenesis will be crucial in order to design molecularly-tailored therapeutic strategies. Alteration of ERG, ETV1, ETV4, FLI1, SPOP, FOXA1 and IDH1 genes have been described in prostate cancer and are ideal candidate for the development of novel agents.

At present, two different studies as investigating the predictive role of TMPRSS2-ERG gene fusion. The first one is an observational study with an estimated enrolment of 65 patients who have been treated with hormonal and radiation treatment after biochemical failure (NCT02588404). The second one will complete its enrolment in January 
2022 and involves patients with biochemical failure status post salvage or radical radiation therapy (NCT02573636). Interestingly, another observational study enrolling subjects with prostate cancer diagnosed on prostate biopsy who undergo radical prostatectomy at Massachusetts General Hospital is comparing the chromosome translocation status (TMPRSS2 fusion with ETS-related genes ERG, ETV1, ETV4, or ETV5) in circulating tumor cells (CTCs) with the primary tumor (NCT01961713). Furthermore, a phase I study is investigating LY3410738, a novel covalent inhibitor of cancer-associated mutant IDH1, in patients with advanced solid tumors (including prostate cancer) with IDH1 mutations (NCT04521686).

Currently, several PARP inhibitors are under evaluation in patients with prostate cancer, used alone or in combination with other therapeutic approaches (34). Among ongoing trials, a phase II trial is exploring the efficacy and safety of PARP inhibitor niraparib as neoadjuvant therapy in patients with high-risk localized prostate cancer with DDR defects (NCT04030559). Another phase II study is investigating how docetaxel with carboplatin followed by rucaparib camsylate works in patients with metastatic castration resistant and $A T M, B R C A 1$ or $B R C A 2$ gene mutations. Interestingly, a phase III study (MK-7339010/KEYLYNK-010, NCT03834519) is ongoing to compare the combination of anti-Programmed Death-1 agent Pembrolizumab and PARP inhibitor Olaparib with abiraterone acetate or enzalutamide in patients with castration resistant prostate cancer.

Our estimates on future clinical and molecular profiles of prostate cancer present several limitations. Indeed, as other prediction systems, they can be affected by errors and biases compared to real data due to advances in molecular and radiologic diagnostic techniques.

\section{Conclusions}

Predicting cancer incidence may be essential to design specific clinical trials, to improve the allocation of economic resources, and to plan future cancer control programs. Our data provide an estimate of future clinical and molecular profiles of prostate cancer and clearly support the design of clinical trials enrolling patients with specific gene mutations and different disease stages.

\section{Acknowledgments}

Funding: None.

\section{Footnote}

Reporting Checklist: The authors have completed the Narrative Review reporting checklist. Available at http:// dx.doi.org/10.21037/tau-20-1439

Provenance and Peer Review: This article was commissioned by the editorial office, Translational Andrology and Urology for the series "Update on Molecular Classification and Individualized Treatments of Genitourinary Tumors". The article has undergone external peer review.

Conflicts of Interest: All authors have completed the ICMJE uniform disclosure form (available at http://dx.doi. org/10.21037/tau-20-1439). The series "Update on Molecular Classification and Individualized Treatments of Genitourinary Tumors" was commissioned by the editorial office without any funding or sponsorship. AC, LC, ALB and RM served as the unpaid Guest Editors of the series. LC serves as an unpaid editorial board member of Translational Andrology and Urology from Dec 2018 to Nov 2022. The authors have no other conflicts of interest to declare.

Ethical Statement: The authors are accountable for all aspects of the work in ensuring that questions related to the accuracy or integrity of any part of the work are appropriately investigated and resolved.

Open Access Statement: This is an Open Access article distributed in accordance with the Creative Commons Attribution-NonCommercial-NoDerivs 4.0 International License (CC BY-NC-ND 4.0), which permits the noncommercial replication and distribution of the article with the strict proviso that no changes or edits are made and the original work is properly cited (including links to both the formal publication through the relevant DOI and the license). See: https://creativecommons.org/licenses/by-nc-nd/4.0/.

\section{References}

1. Available online: http://www.cancer.org/research/ cancerfactsstatistics/cancerfactsfigures2020; (accessed 22th October 2020).

2. Fiorica PN, Schubert R, Morris JD, et al. Multi-ethnic transcriptome-wide association study of prostate cancer. PLoS One 2020;15:e0236209.

3. Kenfield SA, Stampfer MJ, Chan JM, et al. Smoking and prostate cancer survival and recurrence. JAMA 
2011;305:2548-55.

4. Bandini M, Gandaglia G, Briganti A. Obesity and prostate cancer. Curr Opin Urol 2017;27:415-21.

5. Santoni M, Cimadamore A, Massari F, et al. Key Role of Obesity in Genitourinary Tumors with Emphasis on Urothelial and Prostate Cancers. Cancers (Basel) 2019;11:1225.

6. Montironi R, Cimadamore A, Lopez-Beltran A, et al. Morphologic, Molecular and Clinical Features of Aggressive Variant Prostate Cancer. Cells 2020;9:1073.

7. Santoni M, Piva F, Scarpelli M, et al.The origin of prostate metastases: emerging insights. Cancer Metastasis Rev 2015;34:765-73.

8. Ciccarese C, Santoni M, Massari F, et al.Metabolic Alterations in Renal and Prostate Cancer. Curr Drug Metab 2016;17:150-5.

9. Tartari F, Santoni M, Burattini L, et al. Economic sustainability of anti-PD-1 agents nivolumab and pembrolizumab in cancer patients: Recent insights and future challenge. Cancer Treat Rev 2016;48:20-4.

10. Montironi R, Santoni M, Tartari F, et al. Testing PD-1/ PD-L1 Expression in Cancer Therapy: Pathologic Insights and Economic Sustainability. Arch Pathol Lab Med 2016;140:501-2.

11. Tartari F, Conti A, Cerqueti R. Assessing the relationship between toxicity and economic cost of oncological target agents: A systematic review of clinical trials. PLoS One 2017;12:e0183639.

12. Piva F, Tartari F, Giulietti M, et al. Predicting future cancer burden in the United States by artificial neural networks. Future Oncol 2021;17:159-68.

13. Santoni M, Scarpelli M, Mazzucchelli R, et al. Current Histopathologic and Molecular Characterizations of Prostate Cancer: Towards Individualized Prognosis and Therapies. Eur Urol 2016;69:186-90.

14. Ciccarese C, Santoni M, Brunelli M, et al. AR-V7 and prostate cancer: The watershed for treatment selection? Cancer Treat Rev 2016;43:27-35.

15. Cancer Genome Atlas Research Network. The Molecular Taxonomy of Primary Prostate Cancer. Cell 2015;163:1011-25.

16. Cimadamore A, Lopez-Beltran A, Massari F, et al. Germline and somatic mutations in prostate cancer: focus on defective DNA repair, PARP inhibitors and immunotherapy. Future Oncol 2020;16:75-80.

17. Messina C, Cattrini C, Soldato D, et al. BRCA Mutations in Prostate Cancer: Prognostic and Predictive Implications. J Oncol 2020;2020:4986365.
18. Paller CJ, Antonarakis ES. Management of biochemically recurrent prostate cancer after local therapy: evolving standards of care and new directions. Clin Adv Hematol Oncol 2013;11:14-23.

19. Scher HI, Solo K, Valant J, et al. Prevalence of prostate cancer clinical states and mortality in the United States: Estimates using a dynamic progression model. PLoS One 2015;10:e0139440.

20. Sweeney CJ, Chen YH, Carducci M, et al. Chemohormonal Therapy in Metastatic Hormone-Sensitive Prostate Cancer. N Engl J Med 2015;373:737-46.

21. Fizazi K, Tran N, Fein L, et al. Abiraterone plus Prednisone in Metastatic, Castration-Sensitive Prostate Cancer. N Engl J Med 2017;377:352-60.

22. Armstrong AJ, Szmulewitz RZ, Petrylak DP, et al. ARCHES: A Randomized, Phase III Study of Androgen Deprivation Therapy With Enzalutamide or Placebo in Men With Metastatic Hormone-Sensitive Prostate Cancer. J Clin Oncol 2019;37:2974-86.

23. Chi KN, Agarwal N, Bjartell A, et al. Apalutamide for Metastatic, Castration-Sensitive Prostate Cancer. N Engl J Med 2019;381:13-24.

24. Mollica V, Di Nunno V, Cimadamore A, et al. Molecular Mechanisms Related to Hormone Inhibition Resistance in Prostate Cancer. Cells 2019;8:43.

25. Smith MR, Saad F, Chowdhury S, et al. Apalutamide Treatment and Metastasis-free Survival in Prostate Cancer. N Engl J Med 2018;378:1408-18.

26. Small EJ, Saad F, Chowdhury S, et al. Apalutamide and overall survival in non-metastatic castration-resistant prostate cancer. Ann Oncol 2019;30:1813-20.

27. Hussain M, Fizazi K, Saad F, et al. Enzalutamide in Men with Nonmetastatic, Castration-Resistant Prostate Cancer. N Engl J Med 2018;378:2465-74.

28. Sternberg CN, Fizazi K, Saad F, et al. Enzalutamide and Survival in Nonmetastatic, Castration-Resistant Prostate Cancer. N Engl J Med 2020;382:2197-206.

29. Fizazi K, Shore N, Tammela TL, et al. Nonmetastatic, Castration-Resistant Prostate Cancer and Survival with Darolutamide. N Engl J Med 2020;383:1040-9.

30. Di Nunno V, Santoni M, Massari F. New Hormonal Agents in Patients with Nonmetastatic Castration-resistant Prostate Cancer: Can We Be Satisfied with an Advantage in Metastasis-free Survival? Eur Urol Oncol 2019;2:471.

31. Di Nunno V, Mollica V, Santoni M, et al. New Hormonal Agents in Patients With Nonmetastatic Castration-

Resistant Prostate Cancer: Meta-Analysis of Efficacy and Safety Outcomes. Clin Genitourin Cancer 2019;17:e871-7. 
32. Pound CR, Partin AW, Eisenberger MA, et al. Natural History of Progression After PSA Elevation Following Radical Prostatectomy. JAMA 1999;281:1591-7.

33. Kirby M, Hirst C, Crawford ED. Characterising the castration-resistant prostate cancer population: a

Cite this article as: Santoni M, Cimadamore A, Massari F, Sorgentoni G, Cheng L, Lopez-Beltran A, Battelli N, Montironi R. Narrative review: predicting future molecular and clinical profiles of prostate cancer in the United States. Transl Androl Urol 2021;10(3):1562-1568. doi: 10.21037/tau-20-1439 systematic review. Int J Clin Pract 2011;65:1180-92.

34. Aurilio G, Cimadamore A, Santoni M, et al. New Frontiers in Prostate Cancer Treatment: Are We Ready for Drug Combinations with Novel Agents? Cells 2020;9:1522. 